\title{
LXXVII. Reply to Professor Kelland's defence of the Newtonian law of molecular action
}

\section{S. Earnshaw M.A.}

To cite this article: S. Earnshaw M.A. (1842) LXXVII. Reply to Professor Kelland's defence of the Newtonian law of molecular action, Philosophical Magazine Series 3, 21:140, 437-444, DOI: 10.1080/14786444208621605

To link to this article: http://dx.doi.org/10.1080/14786444208621605

册 Published online: 01 Jun 2009.

Submit your article to this journal

Џll Article views: 2

Q View related articles $₫$ 
of film as at the commencement, about double the time is required.

The third cause of error may be avoided by operating with vapours of about the same force. In those described, the average time employed in passing to the maximum was generally about half an hour; if that were not taken into consideration, different results might be obtained.

In regard to chlorine, there exists another cause of complication, the affinity which it possesses for water; for when disengaged in the ordinary manner, chlorine carries with it a certain quantity of water which may very much alter the results of the experiment.

No. 1, St. Mary Abbot's Terrace, Kensington.

[To be continued.]

LXXVII. Reply to Professor Kelland's Defence of the Newtonian Laro of Molecular Action. By S. Earnshaw, M. $A$. Cambridge*.

PROFESSOR Kelland's defence of the extension of Newton's law of force to molecular action consists of a critique upon my memoir "On the Nature of Molecular Forces;" and of a reply to my letter which appeared in your Magazine for July (pres. vol. p. 46). I shall therefore for the sake of precision divide what $I$ have to say in answer to his remarks into two corresponding heads.

1. With respect to the critique on my memoir, it is evident that it has been written by the Professor under the notion that my investigations have supposed each particle of the medium, except the one for which the forces are calculated, to be in their respective equilibrium positions. I gather this from the repeated charge he brings against me of drawing dynamical inferences from a statical investigation. Will the Professor point out what step, in that part of my paper which is written against Newton's law, requires that the particles of the medium should be in their equilibrium positions? With the exception of the last article, where it is expressly stated that the particles are in equilibrium, my paper is an investigation of the properties of a vibrating medium, i.e. a medium in a state of vibration. At any instant during the motion of the medium I fix upon a particle and investigate the properties of the forces which urge it at that moment; the other particles meanwhile are supposed to be in the positions which as particles in a state of vibration they had at the instant fixed upon. [Let

* Communicated by the Author. 


\section{Mr. Earnshaw's Reply to Prof. Kelland's Defence}

the Professor point out one link of my argument against Newton's law which violates this supposition.] I find as a result that there is always one direction in which the particle is urged from its position of rest; and therefore, as the motion of the particle in that direction could not be vibratory, Newton's law cannot be the law of molecular force in the luminiferous æther. This explanation, I trust, will enable the Professor to see that he has written his review of my memoir under the influence of a complete misconception of its nature, to which is due the origin of his complaints that some of my reasonings are unintelligible to him, and that the whole line of my argument is inadmissible (August, p. 130), to which charges it is obviously not necessary for me to make any further reply. There is, however, one argument, which though it belongs to this head, I cannot allow to pass without more particular notice, because upon reading it I could not but consider it as a strong indication of the Professor's having allowed other motives than "a desire for truth" (Sept. p. 207) to influence him in bringing it forward. It stands in the Magazine for this month (p. 270) in these words: "I will only add, when it is concluded from the hypothesis of a cubical arrangement of the particles, acting by forces which vary according to the Newtonian law, that the direction of one side of the cube is stable and of one unstable, ought we not to ask, Is it the hypothesis, or the reasoning based on it which is erroneous? Must it not of necessity be the latter?" Now one would think from the manner in which this argument is brought forward that the matter animadverted upon by the Professor forms a part of my reasoning. Your readers therefore will be surprised to be informed that it stands in my memoir as a purely casual observation, upon which not a step nor even a word of my reasoning against Newton's law depends. Why then did the Professor bring it forward and draw from it the sweeping inference that my reasoning is erroneous? Unfortunately for the Professor, in this instance he reaps no advantage by stepping out of the line of legitimate argument, as his objection is founded on the misconception that $I$ have supposed the particles to be in equilibrium.

2. In commencing his reply to my letter printed in your Magazine of July, the Professor calls upon me to state " what I conceive to be the direct effect of matler." I conceive it to be that effect which arises from the supposition that matter and ather act upon each other by attraction or repulsion (en passant, I do not see why I am called upon for this definition, as I have nowhere employed the direct action of matter). By the indirect action of matter I mean that effect which results 
when the density and arrangement of the æther are changed by the introduction of particles of matter which exclude some particles of æther from certain portions of space, and thereby affect the equilibrium positions of the remaining particles of æther. The Professor next endeavours to guess the reason why I did not draw from my equations any inferences respecting the direct action of matter. The answer is simple; a most important step required that the vibrating medium should consist of homogeneous particles. The step I allude to is that where (S. 3. vol. xx. May, p. 372) I have "'assumed the law of displacement at the time $t$ to be $\xi_{r}=a \sin (r h+\mathrm{T})$," which assumption is not true when the particles of matter vibrate, because then $a$ could not be invariable through the medium. I think no blame attaches to me for causing this perplexity to the Professor, as I have expressly added, "it will be understood that what follows applies only to media in which this law of disturbance can be transmitted," which I understand to be a formal renunciation of all connexion with the direct action of matter.' The Professor, therefore, in referring me to Mr. O'Brien (October, p. 269) to be set right in my notions, seems to have fallen into the mistake of supposing that what Mr. O'Brien has written on the direct effect of matter (March, note at p. 208) can refute what I have written on the indirect effect of matter.

I do not think I fully understand on what ground the Professor affirms (October, p. 264) that I have not taken account of " the roant of action of particles of æther in the portion of space occupied by the material particles" (October, p. 264); for, as I have taken into account all those particles of æther which do exist, and none else that I am aware of, I suppose I must have omitted those which do not exist. Perhaps the Professor will point out what step of my investigation implies the existence of the absent particles.

I am next accused (p. 264) of not saying a word about " the pressure of the particles of matter on the adjacent particles of æther tending to stop their motion." In reply it seems sufficient to state, that the particles of matter are supposed to be so few in number in comparison with the particles of æther in a refracting medium, that though a wave may in some degree be broken up in its passage through the medium by material impediments, the sensible properties of its general front will remain almost, if not entirely unaffected; wherefore in an argument based on the broad features of refraction, any allusion to this consideration were a useless refinement, a needless entering upon difficulties, and an unnecessary inter- 
ruption of my investigations ; which reasons will, I trust, prove satisfactory to the Professor for its having been passed over in silence.

The quotation which the Professor gives at the bottom of p. 265 from my letter (April) I can assure him was not intended to have any reference to his writings. The Professor must also have mistaken my views when he states (p. 266) that I "appear to look for a complete explanation of dispersion to the very quarter at which I aim my objections," for I look to the direct action of matter, against which I have not as yet brought forward any objection.

In the middle of p. 266 the Professor begins his reply to my remarks on his defence of his numerical calculations. It appears to me that he is hereupon somewhat inconsistent with himself. For (May, p. 378) his words are, "my calculations are affected with an error, in that I have neglected to shorten $\lambda$; " but here he writes, "the data are not erroneous." These two statements seem hardly reconcilable. Also, if " the calculations are affected with an error," I do not comprehend how they can "strengthen theory." What he states (p. 267) about his "formula admitting as many arbitrary constants as you please," amounts to a confession that he employed the common principles of interpolations, instead of theory, which is all $I$ have contended for in this part of the subject.

The latter part of the Professor's letter is employed in controverting my remarks on his proof of the transversality of vibrations. The values of $v v^{\prime} u^{\prime \prime}$ which the Professor makes use of in establishing this principle are derived from the equations of motion, which in my last letter $I$ have proved to be nonexistent. That letter is therefore a sufficient answer to this part of the Professor's reply. I cannot, however, dismiss the subject without remarking, that the non-existence of normal vibrations is not proved when it has been shown that (v) the velocity of their transmission is imaginary. It must be shown that $v$ is zero, or very much greater or very much less than the velocity of transmission of the transversal vibrations. For, if it turn out that $v$ is imaginary, the proper inference is, as I have before stated, that the equations of motion have been incorrectly integrated, and the whole investigation needs to be revised. As the remarks which $I$ have made in my last letter respecting the evanescence of the quantity $n$, and, with it, of the equations of motion extend to all that the Professor has written in his Memoirs on Light, and in his Theory of Heat, as far as they are respectively dependent on Newton's law of mole- 
cular action, it is needless to enter further upon the inferences from them which the Professor in various parts of his letters has placed in opposition to my results.

It now only remains to reply to the accusation (p. 267) that I have fallen into an error in turning the equations of motion into that form, from which I drew all my inferences. I can assure the Professor that I did not lay my investigations before the public, without having first carefully revised them, compared them with what other persons have written on the same subject, and satisfied myself as to the cause of differençe where any existed. The Professor may therefore for the future take it for granted that I have seen and examined the equations in M. Cauchy's Mémoire sur la Dispersion de la Lumiere, to which he refers me for correction. I fear it will give to my letter an air of great sameness if $I$ again accuse the Professor of misunderstanding what he has undertaken to criticise. I shall not, however, make the charge without bringing forward the proof of it. The Professor tells me that the coefficient of a certain term of my equations differs in appearance from the corresponding coefficient in $\mathbf{M}$. Cauchy's equations; and his inference is, therefore these coefficients are not equal, and therefore mine are erroneous. Now I ask, how does the Professor know that these coefficients are not equal? I admit that they appear to the eye to be different, but the symbol $\Sigma$ in M. Cauchy's differs entirely from the same symbol in mine. M. Cauchy's coefficients have been brought into the state referred to by reductions suggested by theoretical considerations; but my coefficients were brought into the state in which I leave them by reductions effected upon experimental grounds. If M. Cauchy's differ in value from mine they disagree with experiment, and are therefore to be rejected, as will be made manifest by the following process, which applies equally to $\mathbf{M}$. Cauchy's equations and mine own. But I will first state the matter in another way. In my investigations (March, p. 372), A represents the value of $\Sigma\left\{m^{l} d_{x^{2}}{ }^{2} \mathrm{~F}(\mathrm{R})\right\}$, the summation represented by $\Sigma$ extending to all particles in the $r$ th wave surface, and in all other surfaces the particles of which are in the same state of displacement as in the $r$ th. Also $A$ represents the value of $2 \Sigma\left(A_{r} \sin ^{2} \frac{r h}{2}\right), \Sigma$ now denoting summation for all the values of $r$ in one wave's length. The limiting value of $r$ in performing the operation $\Sigma$ is therefore the number of particles in a wave's length, which number in any conceivable geometrical arrangement of the particles depends upon the 
position of the wave's front. Hence $A_{r}$ and $\sin ^{2} \frac{r h}{2}$ depend upon the direction of transmission; but does $\mathrm{A}$, i.e. $2 \Sigma\left(A_{r} \sin ^{2} \frac{r h}{2}\right)$, also depend upon the direction of transmission? This question, and a similar one for each of the other coefficients, M. Cauchy has not answered, but I have answered it for myself in the negative on experimental grounds, as follows. My equations of motion (and they are M. Cauchy's also) are,

$$
\begin{aligned}
& d_{t}^{2} \xi=-\mathrm{A} \xi-\mathrm{F} \eta-\mathrm{E} \zeta \\
& d_{t}^{2} \eta=-\mathrm{F} \xi-\mathrm{B} \eta-\mathrm{D} \zeta \\
& d_{t}^{2} \zeta=-\mathrm{E} \xi-\mathrm{D} \eta-\mathbf{C} \zeta .
\end{aligned}
$$

The question is, are the coefficients dependent on the position of the wave's front? Multiply these equations respectively by $\cos \alpha, \cos \beta, \cos \gamma$, and add the results, at the same time assuming $k^{2}=\mathrm{A}+\mathrm{F} \frac{\cos \beta}{\cos \alpha}+\mathrm{E} \frac{\cos \gamma}{\cos \alpha}=\mathrm{B}+\mathrm{D} \frac{\cos \gamma}{\cos \beta}$ $+\mathrm{F} \frac{\cos \alpha}{\cos \beta}=\mathrm{C}+\mathrm{E} \frac{\cos \alpha}{\cos \gamma}+\mathrm{D} \frac{\cos \beta}{\cos \gamma}$; from which eliminating $\cos \alpha, \cos \beta, \cos \gamma$, we find the following cubic in $k^{2}$,

$$
\begin{gathered}
\left(k^{2}-\mathbf{A}\right)\left(k^{2}-\mathrm{B}\right)\left(k^{2}-\mathbf{C}\right)-\mathbf{D}^{2}\left(k^{2}-\mathbf{A}\right)-\mathbf{E}^{2}\left(k^{2}-\mathrm{B}\right) \\
-\mathbf{F}^{2}\left(k^{2}-\mathbf{C}\right)=2 \mathrm{D} \mathrm{E} \mathrm{F} .
\end{gathered}
$$

Having from this found three roots $k_{1}^{2}, k_{2}^{2}, k_{3}^{2}$, we can then find three corresponding sets of values of $\cos \alpha, \cos \beta, \cos \gamma$; and our equations of motion by this process of mere algebra take the following simple forms,

$$
d_{t}^{2} \xi^{\prime}=-k_{1}^{2} \xi^{\prime}, \quad d_{t}^{2} \eta^{\prime}=-k_{2}^{2} \eta^{\prime}, \quad: d_{t}^{2} \xi^{\prime}=-k_{s}^{2} \xi^{\prime} ;
$$

where

$$
\begin{aligned}
& \xi^{\prime}=\xi \cos \alpha_{1}+\eta \cos \beta_{2}+\zeta \cos \gamma_{1} \\
& \eta^{\prime}=\xi \cos \alpha_{2}+\eta \cos \beta_{1}+\xi \cos \gamma_{2} \\
& \xi^{\prime}=\xi \cos \alpha_{3}+\eta \cos \beta_{3}+\zeta \cos \gamma_{3},
\end{aligned}
$$

that is, $\xi^{\prime} \eta^{\prime} \zeta^{\prime}$ are the displacements of the particle $n$ estimated parallel to a new set of rectangular axes. The forms of the new equations of motion show that these axes are axes of dynamical symmetry,-those in fact which are better known as the axes of elasticity. Now from experiment we know that for waves of a given length $k_{1}{ }^{2}, k_{2}{ }^{2}, k_{3}{ }^{2}$ are constant quantities, i. e. independent of the position of the waves' front (by the above process I have only changed the axes of coordinates, the waves' front remains unaltered in position). And not to occupy room unnecessarily, I now refer the Professor to the note (July, p. 4.8) to my letter for the remainder of the proof that " $A, B, C, D, E, F$ are independent of the position of the 
wave's front." By this process it is established beyond the possibility of a doubt, that when the operation represented by $\Sigma$ is performed in the expression which Professor Kelland quotes (p. 268) from M. Cauchy, the result ought to be independent of the position of the wave's front; and so it is proved either that my equations and M. Cauchy's are identical, or that M. Cauchy's are at variance with experiment. The methods by which we have obtained our equations are perfectly dissimilar, but I believe the equations themselves are identical. In deducing his M. Cauchy has adhered closely to theoretical considerations; but in deducing mine, I have proceeded to a certain point by the guidance of theory, and then beginning from a more advanced point, where the results of experiment were known, have worked backwards to meet theory. It is therefore easily seen that my results being a mixture of theory and experiment would not present the same appearance to the eye as the results of M. Cauchy, which are obtained from theory alone. They must, however, be identical in fact, or else theory is discordant with experiment. What therefore Professor Kelland has written (p. 268) about "the axis of transmission" is grounded on a misconception, from which also has sprung his idea that "the form of my equations" (p. 46), from which my inferences have been drawn against the Newtonian law, \&c., " does depend on the position of the front of the wave."

I believe I have now replied to every objection of importance which Professor Kelland has brought forward; I cannot however conclude this letter without remarking, that it is obvious that a discussion like the one in which we are now engaged never can be brought to a satisfactory conclusion unless both parties write with perfect candour and a single eye to the discovery of the truth. All arguments which do not really bear upon the Newtonian law must be avoided; and those which do bear upon it, if after due scrutiny they be found to be true, unhesitatingly admitted with all their consequences. I would therefore, with a view of shortening our labours, respectfully request the Professor not to take so wide a field, but to confine himself to the prominent and really important points of the argument; because if objections of this character cannot be answered, it is clearly quite unnecessary for him to descend with M. Cauchy into the mystical and doubtful subtilties of "refined analysis." May I then respectfully requesi the Professor to answer in the spirit here recommended the four following queries, which seem to me better calculated than any others to bring our discussion to a speedy termination?- 
444. Dr. Booth on a Theorem in Analytical Geometry.

1. Does Professor Kelland admit that I have satisfactorily proved that the quantity $n$ used in his memoir on dispersion is equal to zero?

2. Does he admit that the evanescence of that quantity destroys his equations of motion?

3. Does he admit that the evanescence of his equations of motion destroys his proof of the transversality of vibrations?

4. Does he admit that the disappearance of his equations of motion in a medium of perfect symmetry rohenever Neroton's law is introduced, is a sufficient proof that that cannot be the law of molecular action?

If he does admit these points our discussion is at an end; but if he does not, I shall with great willingness answer any objections against these which he may think it necessary to bring forward. The introduction of collateral questions (such as, "whether the force acts by attraction or repulsion," " whether a cubical arrangement is or is not one of geometric symmetry," "whether the æther has boundaries," "how vibrations are generated," "whether it is probable that a violent effort would be requisite to move a particle of æther out of its position of equilibrium," and others of a similarly discursive nature which the Professor has mooted in his letters) tends unnecessarily to distract attention from the main question; they may therefore safely be allowed by both parties to stand over as unimportant till all objections which are of the first magnitude have been refuted or allowed.

Cambridge, Oct. 7, 1842.

LXXVIII. On a Theorem in Analytical Geometry. By the Rov. JaMes Booth, LL.D., M.R.L.A.

[Continued from p. 179.]

HAVING shown that if three fixed points assumed on a right line are always retained in three fixed planes, any fourth point $\mathbf{P}$ will describe an ellipsoid, whose centre is the common intersection of the three planes, we proceed to establish the following remarkable property, that the volume of this ellipsoid is independent of the angles between the coordinate axes; a singular result, to which an analogous property may be found in the ellipse.

Resuming the equation found at page 178 ,

$$
\mathrm{V}=\frac{x^{2}}{a^{2}}+\frac{y^{2}}{b^{2}}+\frac{z^{2}}{c^{2}}+\frac{2 \cos \lambda}{b c} y z+\frac{2 \cos \mu}{a c} x z+\frac{2 \cos \nu}{a b} x y-1=0 .
$$

When the equation of the ellipsoid is in this form, having all its terms positive, the point $\mathrm{P}$ is supposed to be external to 